\title{
ON THE INFINITE IN MEREOLOGY WITH PLURAL QUANTIFICATION
}

\author{
MASSIMILIANO CARRARA and ENRICO MARTINO \\ University of Padua
}

\begin{abstract}
In "Mathematics is megethology," Lewis reconstructs set theory using mereology and plural quantification (MPQ). In his recontruction he assumes from the beginning that there is an infinite plurality of atoms, whose size is equivalent to that of the set theoretical universe. Since this assumption is far beyond the basic axioms of mereology, it might seem that MPQ do not play any role in order to guarantee the existence of a large infinity of objects. However, we intend to demonstrate that mereology and plural quantification are, in some ways, particularly relevant to a certain conception of the infinite. More precisely, though the principles of mereology and plural quantification do not guarantee the existence of an infinite number of objects, nevertheless, once the existence of any infinite object is admitted, they are able to assure the existence of an uncountable infinity of objects. So, if-as Lewis maintains-MPQ were parts of logic, the implausible consequence would follow that, given a countable infinity of individuals, logic would be able to guarantee an uncountable infinity of objects.
\end{abstract}

§1. Introduction. In "Mathematics is megethology" Lewis (1993) reconstructs set theory using mereology and plural quantification (hereafter: MPQ). Lewis assumes from the beginning that there is an infinite plurality of atoms, whose size is equivalent to that of the set theoretical universe. So, it might seem that MPQ, though they supply a suitable language for reconstructing set theory, nevertheless they do not play any role in order to guarantee the existence of a large infinity of objects.

On the contrary, we intend to demonstrate that MPQ are, in some ways, particularly relevant to a certain conception of the infinite. More precisely, though the principles of MPQ do not guarantee the existence of an infinite number of objects, nevertheless, once the existence of any infinite object is admitted, they are able to assure the existence of an uncountable infinity of objects. ${ }^{1}$

We divide our paper into the following sections. In Section 2, we will briefly review the crucial assumptions underlying Lewis' reconstruction of set theory. In Section 3 we will construct a mereological model of the power set of any plurality of pairwise nonoverlapping individuals and show that any infinite individual has uncountably many parts. In Section 4, we will make some final remarks on the alleged innocence of MPQ, on its significance for the conception of the infinite and on the problem of impredicativity of the uncountably infinite.

§2. Lewis' megethology. "Mereology," literally the "science of parts," analyzes the relation "... is a part of ...." There are different formulations of mereology depending on

Received: December, 22, 2009

1 For an analysis of the power of MPQ see also Geoffrey Hellman's development of modalstructuralism in Hellman (1996). 
the language adopted (for a general introduction see Simons, 2000, and Burkhardt et al., 2009). Since we intend to consider Lewis' reconstruction of set theory with mereology, we will utilize his formulation, which is suited to the relevant aspects of the problem we are analyzing. Lewis treats mereology in a plural language, a language extending that of first-order logic, including singular and plural reference and singular and plural quantification (for a modified proposal of megethology see Martino, 1996). Plural quantification is a reinterpretation of the second-order monadic logic proposed by Boolos $(1984,1985)$. In Boolos' perspective second-order monadic logic is ontologically innocent. Contrary to the most accredited view, it does not entail any commitment to classes or to properties but only to individuals, as first-order logic does. According to Boolos' interpretation, secondorder quantification differs from first-order only in that it refers to individuals plurally, while the latter refers to individuals singularly.

By combining mereology with plural quantification, Lewis introduces megethology, a powerful framework in which one can formulate strong assumptions about the size of the universe of individuals (corresponding to the existence of strongly inaccessible cardinals). Within this framework, Lewis develops a structuralist class theory, in which the role of classes is played by certain fusions of atoms and the membership relation is defined in mereological terms.

Lewis' megethology can be formalized into a first-order language with identity with two sorts of variables:

Singular variables: $x, y \ldots$

Plural variables: $X, Y \ldots$

We introduce the primitive nonlogical constants:

$\leq: x \leq y$ is to be read " $x$ is part of $y$ ";

$\eta: x \eta X$ is to be read " $x$ is one of $X$ s."

We define:

$x<y$ (x is a proper part of $\mathrm{y})$ if $_{d f}(x \leq y \wedge x \neq y)$;

$x$ is an atom if f $_{d f}$ it has no proper parts;

$X \sqsubseteq Y$ (the $X$ s are among the $Y \mathrm{~s})$ if $_{d f} \forall x(x \eta X \rightarrow x \eta Y)$;

$X \sqsubset Y$ (the $X \mathrm{~s}$ are among, but not all, the $Y \mathrm{~s})$ if $_{d f}(X \sqsubseteq Y \wedge X \neq Y$ );

$X \sqcup Y$ (the sum of the $X \mathrm{~s}$ and the $Y \mathrm{~s})$ is the plurality $Z$ such that $\forall x(x \eta Z \leftrightarrow x \eta X \vee x \eta Y)$;

$x \circ y(x$ overlaps $y)$ if $_{d f} \exists z(z \leq x \wedge z \leq y)$

$y$ is a sum of the $X \mathrm{~s}$, in symbols $\sigma X$, if $d f$ each of the $X \mathrm{~s}$ is a part of $y$ and each part of $y$ overlaps one of the $X$ s. Formally:

$\forall x(x \eta X \rightarrow x \leq y) \wedge \forall x(x \leq y \rightarrow \exists z(z \eta X \wedge x \circ z)$

$\pi X$ (the product of the $X \mathrm{~s}$ ) is the fusion of all common parts of all the $X \mathrm{~s}$, provided there is at least one part common to all the $X \mathrm{~s}$.

We will first introduce the axioms for mereology:

AXIOM 2.1. $\leq$ is a partial order (reflexive, antisymmetric, transitive).

Aхіом 2.2 (АТомісіту). Every individual has an atomic part.

AXIOM 2.3. For every $\mathrm{X}$ there is a unique sum of the $\mathrm{X}$ s.

From Axioms 2.2 and 2.3 it straightforwardly follows that every individual is the fusion of its atoms.

Besides, the crucial comprehension for pluralities: 
PCA 2.4. Given any formula $\phi$ of our language, satisfied by at least an individual, there is the plurality of all individuals satisfying it:

$\exists x \phi \rightarrow \exists X \forall x(x \eta X \leftrightarrow \phi)$

Finally, we list Lewis' definitions and axioms concerning the size of the universe.

Definitions:

An individual is small if $f_{d f}$ there is no bijection between its atoms and all atoms of the universe $V$;

A plurality $X$ is small if if $_{d f}$ there is no bijection between the $X$ s and all atoms of the universe $V$;

An individual $x$ is infinite if $_{d f}$ there is a bijection between its atoms and the atoms of a proper part of $x$.

Axioms:

AXIOM 2.5. The fusion of few small things is small.

AхIOM 2.6. The parts of a small thing are few.

AXIOM 2.7. Something small is infinite.

§3. Uncountable infinity. Lewis' axioms on the size of the universe are very strong. They assure the existence of so large an infinity of atoms that it is adequate to represent all sets of the usual set theory. ${ }^{2}$ In particular, from the assumptions:

Aхіом 2.2 (АТОмісіту). Every individual has an atomic part,

\section{AXIOM 2.7. Some small individual is infinite}

it follows immediately that the universe of atoms is uncountable.

Although the foregoing axioms are expressible in the language of mereology and pluralities, they are not concerned with either in the sense that they are extraneous to the characterization of both mereology and pluralities. Therefore, they fail to bring to light how the combination of mereology and pluralities affects the concept of the infinite. Of course, such a combination fails to guarantee the existence of infinitely many individuals. However, we want to show that, under the assumption of an infinity of individuals, mereology, with or without atoms, and plural quantification are able to guarantee the existence of an uncountable infinity of individuals.

We begin by presenting an informal exposition of the argument based on the intuitive notion of infinite, in particular of the countable infinite. Subsequently, we will bring forward the axioms of mereology and plural quantification that are suitable for the formalization.

LEMMA 3.1. If there are infinitely many individuals, then there are infinitely many pairwise nonoverlapping individuals.

Proof. Suppose there are infinitely many individuals. We distinguish two cases:

Case 1) Every individual has an atomic part.

Since every individual is the fusion of its atoms, there are are infinitely many atoms, which are obviously pairwise nonoverlapping.

${ }^{2}$ Lewis himself shows that from his axioms follows that "the small parts of reality are barily many" (in Lewis, 1993, p. 19). This means that the small parts of reality (which play the role of sets) are as many as the totality of atoms. 
Case 2) Some individuals have no atomic part.

Let $x$ be such an individual. Then there is a countable infinity of individuals $x_{0}, x_{1} \ldots$, $x_{\mathrm{n}} \ldots$ where $x_{0}=x$ and, for all $n \in \mathbb{N}, x_{\mathrm{n}+1}$ is a proper part of $x_{n}$. The relative complements, $x_{0}-x_{1}, \ldots x_{n}-x_{n+1}, \ldots$, are pairwise nonoverlapping.

THEOREM 3.2. Let X be a countable plurality of pairwise nonoverlapping individuals $x_{0}, x_{1} \ldots, x_{n} \ldots$ The plurality $Y$ of all fusions of some of the Xs is uncountable.

Proof. Suppose, by reduction, that there are only countably many $Y \mathrm{~s}: y_{0}, y_{1} \ldots, y_{n} \ldots$ Let $y$ be the fusion of $x_{0}$ and all $x_{n}$ that are not part of $y_{n-1}$ (for $n>1$ ). Since, for all $n, x_{n+1}$ is a part of $y$ iff it is not part of $y_{n}$, it follows that, for all $n, y \neq y_{\mathrm{n}}$.

It follows that, provided there are infinitely many individuals, there are uncountably many individuals.

We want to show that our argument rests on very intuitive axioms of mereology and plural quantification. To the purpose we will reformulate the axioms and definitions of MPQ, as well as the definition of infinite, as follows.

AXIOM 3.3. $\leq$ is a weak partial order (reflexive, antisymmetric, transitive).

AXIOM 3.4. For any plurality $X$ of individuals, there is a unique individual $x$ such that each individual overlaps $x$ iff it overlaps at least one of the Xs. $x$ is the sum (or fusion) of the Xs, in symbols $x=\sigma X$.

The sum of two individuals $x, y$ will be indicated by $x+y$.

COROLLARY 3.5. If $x$ is a proper part of $y$, then some part of $y$ does not overlap $x$.

Proof. Suppose, by way of contradiction, that every part of $y$ overlaps $x$. Then both $x$ and $y$ are the sum of the plurality formed by $x$ alone, against the uniqueness of the sum.

AXIOM 3.6. If there is a part common to all Xs, then there is an $x$ such that, for all $y, y$ is a part of $x$ iff it is part of each one of the Xs. Such an $x$, necessarily unique, is the product of the Xs, in symbols $x=\pi X$. The product of two individuals $x$ and $y$ will be indicated by $x \cdot y$.

THEOREM 3.7. $\sigma X$ is the least upper bound of the Xs, that is, for all $y, \sigma X$ is part of $y$ iff each of the Xs is part of $y$.

Proof. Suppose that each of the $X \mathrm{~s}$ is part of $y$. Assume, by reduction, that $\sigma X$ is not part of $y$. Since $y$ overlaps all $X \mathrm{~s}, \sigma X$ overlaps $y$. So $y \cdot \sigma X$ is a proper part of $\sigma X$ and, by Corollary 3.5, there is a part $z$ of $\sigma X$ that does not overlap $y \cdot \sigma X$, whence it does not overlap $y$. However, since $z$ overlaps $\sigma X$, it must overlap one of the $X \mathrm{~s}$, which is absurd because the $X$ s are parts of $y$. So $\sigma X$ is part of $y$.

Vice versa let $\sigma X$ be a part of $y$. Suppose, by reduction, that some $x$ is such that $x \eta X$ and $x \not \leq y$. By Corollary 3.5 there is a $z \leq x$ nonoverlapping $y$; but this is absurd, because $z$ must overlap $\sigma X$ which is part of $y$.

THEOREM 3.8. If $y$ is not part of $x$, then there is a unique $z$ such that $x$ and $z$ are nonoverlapping and $y=x \cdot y+z$ (if $x$ and $y$ are nonoverlapping, $y=z$ ). $z$ is the relative complement of $x$ in $y$ and is indicated by $y-x$.

Proof. Suppose that $x$ overlaps $y . x \cdot y$ is a proper part of $y$. So there is some part of $y$ which does not overlap $x \cdot y$. Let $z$ be the the sum of all the parts of $y$ nonoverlapping $x \cdot y$. So $z$ is a part of $y$ which does not overlap $x \cdot y$. Suppose, by reduction, that $x \cdot y+z$ 
is a proper part of $y$. Then there is a part $u$ of $y$ which does not overlap $x \cdot y+z$ and therefore does not overlap $x \cdot y$. By construction of $z$, $u$ overlaps $z$, so $u$ overlaps $x \cdot y+z$, contradiction

Besides, we will exploit the crucial axiom of pluralities, the comprehension principle:

AXIOM 3.9 (COMPREHENSION PRINCIPLE). The individuals satisfying any formula of the formal language (satisfied by at least one individual) form a plurality.

We do not assume the notion of ordered pair as primitive in order to mereologically reproduce the notion of function. Instead, we will define a restricted notion of ordered pair, suitable for our purposes, in mereological terms, without introducing any axiom ad hoc.

DEFINITION 3.10. An individual a is infinite if there is a plurality $X$ of parts of a such that, for all $x \eta X$, there is a $y \eta X$ such that $y<x$.

DeFinition 3.11. Let $X$ be a plurality and let $x \eta X, y \eta X . y$ is a close $X$-part of $x$, in symbols $y \ll_{X} x$, if $y<x$ and there is no $z \eta X$ such that $y<z<x$.

AXIOM 3.12 (CHOICE). Let $X$ be a plurality such that, for all $x \eta X$, there is a $y \eta X$ such that $y<x$. Then there is a plurality $Y \sqsubseteq X$ such that, for all $y \eta Y$, there is a unique $z \eta Y$ such that $z \ll Y y^{3}$

Definition 3.13. Two pluralities $X, Y$ are separate if no $x \eta X, y \eta Y$ are overlapping. If $X$ and $Y$ are separate, an $X-Y$-pair is the sum $x+y$ of an $x \eta X$ and $a y \eta Y . x$ is the $X$-component, $y$ the $Y$-component of the pair.

TheOrem 3.14. Let $X, Y$ be separate. Two $X$-Y-pairs $x+y, x^{\prime}+y^{\prime}$ are identical iff $x=x^{\prime}, y=y^{\prime}$.

Proof. Suppose $x+y=x^{\prime}+y^{\prime}$. We want to show that $x^{\prime} \leq x$. Assume, by reduction, that $x^{\prime} \not \leq x$. Let $z=x^{\prime}-x$. So, $x^{\prime}=x \cdot x^{\prime}+z$. Since $z \leq x^{\prime}+y^{\prime}=x+y, z$ must overlap $x$ or $y$; but it cannot overlap $x$ because $z$ is the relative complement of $x$ in $x^{\prime}$ and it cannot overlap $y$ because $x^{\prime}$ does not overlap $y$. This is absurd. So, $x^{\prime} \leq x$. Similarly $x \leq x^{\prime}$, whence $x=x^{\prime}$. Analogously $y=y^{\prime}$.

DEFINITION 3.15. A relation between two separate pluralities $X, Y$ is any plurality $R$ of $X$-Y-pairs. A function from $X$ to $Y$ is a relation satisfying the usual conditions of uniqueness. Iff is a function from $X$ to $Y$, we use the familiar notation $f(x)$ to indicate the unique $y$ such that $(x+y) \eta f$. We define injections, surjections, and bijections with the usual restrictions.

Definition 3.16. Let $X, Y$ be separate pluralities. The size of $X$ is identical to the size of $Y$ if there is a bijection of $X$ to $Y$. The size of $X$ is smaller than that of $Y$ if there is an injection but no bijection of $X$ to $Y$.

OBSERVATION 3.17. The set $\mathbb{N}$ of natural numbers satisfies the following condition: there are three subsets of $\mathbb{N} \alpha, \beta, \gamma$ such that

3 Observe that, dealing with arbitrary pluralities of individuals, our axiom of choice is quite evident. For, the $Y \mathrm{~s}$ can be selected from the $X \mathrm{~s}$ by performing infinitely many acts of choice. And it is implicit in the notion of plurality that, however certain individuals are chosen, they form a plurality (not so in set theory, where the mere existence of certain individuals does not guarantee that they form a set). For a discussion of different versions of the axiom of choice in the framework of plural quantification see Pollard (1988). 
(i) $\alpha$ and $\beta$ are disjoint, $\gamma \subset \beta$;

(ii) there is a bijection $f$ of $\alpha$ to $\beta$ and a bijection $g$ of $\alpha$ to $\gamma$. (Take, for instance, $\alpha=$ the set of even numbers, $\beta=$ the set of odd numbers, $\gamma=$ the set of odd numbers $>1, f(n)=n+1, g(n)=n+3)$.

The clauses (i) and (ii) of Observation 3.17, suitable for a characterization of the infinite, suggest the following definition:

DEFINITION 3.18. A plurality $X$ of pairwise nonoverlapping individuals is infinite if there are pluralities $Y, Z, U$ such that:

(i) $Y, Z$ are separate, $Y \sqcup Z=X, U \sqsubset Z$;

(ii) There are bijections $f: Y \rightarrow Z, g: Y \rightarrow U$.

Let $X$ be an infinite plurality of pairwise nonoverlapping individuals and let $Y, Z$, $U, f, g$ be as above. Keeping the meaning of these symbols fixed, we will construct a mereological model of the sets of $Y \mathrm{~s}$.

Let $j \eta(Z-U)$ be an arbitrary fixed individual. Take as a set $j$ alone or the sum of $j$ with some of the $Y$ s. If $\alpha$ is a set and $y \eta Y, y$ is a member of $\alpha$, in symbols $y \in \alpha$, if $d f$. $y \leq \alpha$. Thus, $j$ is the empty set $\varnothing$ and $j+y$ is the singleton $\{y\}$ of $y$.

EXTENSIONALITY 3.19. If two sets $\alpha$ and $\beta$ have the same members, they are identical.

Proof. Suppose that $\alpha$ and $\beta$ have the same members. Since $\alpha$ is the sum of $j$ and (possibly) some of the $Y$ s and these are parts of $\beta$, it follows that $\alpha \leq \beta$. Likewise $\beta \leq \alpha$, whence $\alpha=\beta$.

COMPREHENSION 3.20. Any $Y \mathrm{~s}$ form a set. More precisely, if $H$ is any plurality of $Y \mathrm{~s}$, there is a set $\alpha$ such that, for all $y \eta Y, y \eta H$ iff $y \in \alpha$.

Proof. Let $\alpha$ be the sum of $j$ and the $H$ s. Thus, anyone of the $H$ s is a member of $\alpha$.

Vice versa, if $y \in \alpha$, then $y \leq \alpha$. Hence $y$ overlaps $j$ or one of the $H$ s. As the $Y$ s are pairwise nonoverlapping and $y \neq j, y$ must be one of the $H$ s.

From the comprehension principle for pluralities it follows immediately that:

COROLLARY 3.21. The Ys satisfying any formula of the language form a set.

UNION 3.22. For any plurality $H$ of sets, there is a set $\alpha$ such that, for all $y \eta Y, y \in \alpha$ iff it belongs to some of the $H \mathrm{~s}$. $\alpha$ is the union of the $H \mathrm{~s}$, in symbols $\alpha=\cup H$.

Proof. $\alpha$ is the sum of $j$ and all members of the $H$ s.

We will give a mereological reconstruction of Cantor's theorem.

To the purpose, define the ordered pairs of $Y \mathrm{~s}$ as follows: if $y_{1}, y_{2} \eta Y$, define the ordered pair $\left(y_{1}, y_{2}\right)$ as $y_{1}+g\left(y_{2}\right)$. We can assume, without loss of generality, that there is a $j^{\prime} \eta(Z-U), j^{\prime} \neq j$, and extend $g$ by putting $g(j)=j^{\prime}$. We can now extend in an obvious way the notion of ordered pair to the plurality $Y^{*}$ of all sums of individuals in the plurality $(j \sqcup H)$. So we can recover the notion of function (understood as a plurality of ordered pairs) between any two subpluralities of $Y^{*}$ and we can compare the sizes of such pluralities.

We can now reproduce the well-known proof of Cantor's theorem:

THEOREM 3.23. The plurality $S$ of all sets of $Y$ S is larger than $Y$.

Proof. We will show that there is an injection but no bijection from $Y$ to $S$. 
(i) The plurality of ordered pairs of form $(y,\{y\})$ is the required injection.

(ii) Suppose, by way of reduction, that $h$ is a bijection from $Y$ onto $S$. Let $\alpha=\{y$ : $y \notin h(y)\}$ and let $a \eta Y$ be such that $h(a)=\alpha$. It turns out that $a \in \alpha$ iff $a \notin \alpha$, contradiction. Therefore there can be no such bijection.

DEFINITION 3.24. An infinite subplurality $K$ of $Y^{*}$ is countable if $f_{f}$ it is bijective with any of its infinite subpluralities.

COROLlARY 3.25. S is uncountable.

THEOREM 3.26. If $a$ is infinite, there is an infinite plurality of pairwise nonoverlapping parts of $a$.

Proof. By the axiom of choice, there is a plurality $X$ of parts of $a$ such that, for all $x \eta X$, there is a unique $y \eta X$ such that $y \ll_{X} x$. Let $b \eta X$ and $c \eta X$ be such that $c \ll b$ and define $Y$ as the least plurality satisfying the following clauses:

(i) $(b-c) \eta Y$;

(ii) if $(x-y) \eta Y$, where $y \ll_{X} x$ and $z \ll_{X} y$, then $(y-z) \eta Y$.

It is easily seen that $Y$ is an infinite plurality of pairwise nonoverlapping parts of $a$. For, one can order $Y$ as the natural numbers, taking $(b-c)$ as 0 and $(y-z)$ as the successor of $(x-y)$ and argue as we did for the natural numbers. ${ }^{4}$

From Theorems 3.23, 3.26 it follows that:

THEOREM 3.27. There are uncountably many parts of any infinite individual.

\$4. Concluding remarks. Lewis maintains that mereology is ontologically innocent. In particular, he argues that, unlike set theoretical membership, the existence of the mereological sum of any plurality $X$ of individuals does not commit to the existence of any new individual beyond the $X \mathrm{~s}$. On the other hand, he clearly treats the sum of the $X \mathrm{~s}$ as an outright individual, different (in general) from all $X$ s. In contrast, he does not regard a plurality $X$ as a singular individual (any talk of $X$ as a single entity is to be understood as a mere linguistic device to talk of the $X$ s plurally). "Plural quantification is innocent: we have many things, we do mention one thing that is the many taken together. Mereology is innocent in a different way: we have many things, we do mention one thing that is the many taken together, but this one thing is nothing different from the many" (Lewis, 1991, p. 87).

How can it be that this single thing is nothing different from the many? Of course, Lewis' claim, literally understood, would be contradictory. We think that it is to be understood in the sense that whoever accepts the existence of the $X \mathrm{~s}$, commits oneself automatically to the existence of their sum (for a more detailed discussion of the innocence of mereology see Carrara \& Martino, 2009).

By virtue of Theorem 3.27, this entails that the acceptance of the countably infinite forces one to accept the uncountably infinite as well.

4 Assuming the axiom of atomicity 2.2 one can avoid the axiom of choice. For, since atoms are pairwise nonoverlapping, one can trivially obtain Theorem 3.26 by redefining the infinity of an individual as follows: $a$ is infinite if $_{d f}$ it has infinitely many atoms (according to Definition 3.18). 
However, the history of the philosophy of mathematics seems to show that the claim that any infinite is countable can be held within a respectable conception of mathematics. Such famous advocates as Kronecker, Poincaré and Skolem, among others, have defended this claim. Besides, having recovered the notion of ordered pair for the plurality $S$ of the sets of $Y \mathrm{~s}$, the continuum hypothesis $\mathrm{CH}$ is formulable in our language, so that the standard interpretation of the primitives of MPQ determines whether there are intermediate infinities between the countable and the continuum. The truth or falsity of $\mathrm{CH}$ is a typical set theoretical question that seems to transcend the frame of pure logic. ${ }^{5}$

Thus, the alleged innocence of mereology, in particular Lewis' thesis that mereology is part of logic (see Lewis, 1991, pp. 81-82), seems highly implausible. As already observed in Section 3, the fact that the universe cannot be countably infinite trivially follows from Lewis' axiom of infinity 2.7, according to which some infinite individual is small. But we want to stress again that Axiom 2.7, though expressed in the language of mereology with plural quantification, is an axiom neither of mereology nor of plural quantification. It is an axiom about the size of the universe that assumes that the universe is uncountable. So, even under Lewis' assumption that mereology and plural quantification are part of pure logic, using that axiom to show the existence of an uncountable infinity does not justify the conclusion that the existence of the latter is a logical consequence of the existence of a countable one. In contrast, the interest of our approach is that such conclusion can be justified by a minimal stock of basic axioms concerning only mereology and plural monadic quantification. The severe constraints of the axioms used in our deduction have been essential in order to enlighten the commitment to the infinite implicit in Lewis' alleged logic.

On the other hand, the notion of the infinite supported by MPQ is much weaker than that supported by general set theory. Mereology, unlike set theory, does not guarantee that, given any infinite, there is always a larger infinite. As we saw, mereology assures that, given any infinite plurality, there is also a countable plurality of pairwise nonoverlapping individuals, and that, given any plurality of pairwise nonoverlapping individuals, there exists a larger plurality of individuals. However, there is no evidence that these latter might be pairwise nonoverlapping, so that the reiteration of our procedure for finding a larger plurality is prevented.

This fact supplies a very intuitive solution to Cantor's paradox: the size of the universe of all individuals is larger than that of every plurality of pairwise nonoverlapping individuals. As far as we know, our axioms may be consistent with the conjecture that there are only two sizes of infinite: the countable and the continuum. ${ }^{6}$ If so, MPQ might support an interesting conception of the infinite, intermediate between the countable and the set theoretical.

Finally, observe that the crucial axiom of sums (3.4), far from being ontologically innocent, helps to explicate the very strong ontological assumption underlying the classical

5 We are grateful to an anonymous referee for suggesting this remark.

6 As observed by the referee, according to Field's nominalistic perspective, one can maintain the existence of an infinity of atoms of the cardinality of the continuum, the points of the geometrical space. We think, however, that the geometrical intuition of point is far less clear than that of the countable infinite, as shown, for instance, by Zeno's paradoxes. In fact, the structure of the geometrical space has been clarified through the set theoretical interpretation. We think, therefore, that MPQ is compatible with a conception of infinite that accepts a countable plurality of atoms but rejects geometrical points as primitive entities. 
set theoretical semantics of second-order logic: that any individuals, however chosen, form a set. This assumption is the justification, within a realist conception of objects, of the second-order logic comprehension principle, saving it from the charge of impredicativity. According to that assumption, the existence of a set of individuals is quite independent of any property used in order to isolate its members. Thus, no circularity is involved in describing a set of individuals by quantifying over all sets of individuals.

\$5. Acknowledgment. We would like to thank the referee for the helpful comments and suggestions.

\section{BIBLIOGRAPHY}

Boolos, G. (1984). To be is to be a value of a variable. (Or to be some values of some variables). Journal of Philosophy, 81, 430-449.

Boolos, G. (1985). Nominalist platonism. Philosophical Review, 94, 327-344.

Burkhardt, H., Seibt, J., \& Imaguire, G., editors. (2009). Handbook of Mereology. Munich, Germany: Philosophia Verlag.

Carrara, M., \& Martino, E. (2009). On the ontological commitment of mereology. Review of Symbolic Logic, 2, 164-174.

Hellman, G. (1996). Structuralism without structures. Philosophia Mathematica, 4, $100-123$.

Lewis, D. K. (1991). Parts of Classes. Oxford, UK: Oxford University Press.

Lewis, D. K. (1993). Mathematics is megethology. Philosophia Mathematica, 1, 3-23.

Martino, E. (1996). La teoria degli insiemi in un platonismo nominalista. In Abrusci, V. M., Cellucci, C., Cordeschi, R., and Fano, V., editors. Prospettive della logica e della filosofia della scienza. Pisa, Italy: ETS, pp. 445-451.

Pollard, S. (1988). Plural quantification and the axiom of choice. Philosophical Studies, 54, 393-397.

Simons, P. (2000). Parts: A Study in Ontology. Oxford, UK: Oxford University Press.

\section{DEPARTMENT OF PHILOSOPHY \\ UNIVERSITY OF PADUA \\ PADOVA, ITALY}

E-mail: massimiliano.carrara@unipd.it, enrico.martino@unipd.it 